\title{
Peripheral Nerve Stimulation for Treatment of Painful Osteoarthritis of the Knee: A Case Report
}

\author{
Gustaf Van Acker, MD, PhD ${ }^{1,2}$, Richard D. Wilson, MD, $\mathrm{MS}^{1-3}$, and Chong H. Kim, MD ${ }^{1-3}$
}

Background: Osteoarthritic knee (OAk) pain is common, yet the standard of care often yields unsatisfactory pain relief. There remains a role for novel treatment options. Percutaneous motor peripheral nerve stimulation (mPNS) of the knee is a novel minimally invasive procedure that stimulates motor end points leading to muscle contraction associated with the painful joint. Pain relief is hypothesized to be achieved through central pain modulation.

Case Report: We report the case of a patient who experienced refractory osteoarthritic knee pain after 9 months of conservative care. Following 7 weeks of mPNS treatment, the patient achieved improvement in OAk pain relief and activities of daily living as measured by notable improvements in the Brief Pain Inventory-Short Form and Knee Injury and Osteoarthritis Outcome Score at 8- and 12-weeks postimplant. The Patient Global Impression of Change at the end of stimulation was much improved.

Conclusion: Motor PNS may offer a safe and effective treatment alternative for chronic refractory pain related to OAk.

Key words: Osteoarthritis, knee, pain, peripheral nerve stimulation

\section{BACKGROUND}

Pain due to osteoarthritis is common, with the knee being the most common lower limb joint affected. The prevalence of those experiencing pain due to osteoarthritis of the knee (OAk) is increasing, affecting approximately 9.3 million adults over age 45 in the US $(1,2)$. Many clinical practice guidelines have been published regarding OAk management $(3,4)$. Unfortunately, nonsurgical treatment efficacy remains low, with $41 \%$ of individuals having progressive symptoms over time (3). Additionally, $16-33 \%$ of those who undergo total knee arthroplasty have persistent chronic knee pain that may require additional treatment (5). Given the standard of care shortcomings, there remains a role for novel treatments for chronic pain related to OAk.
We report a case for feasibility, safety, and potential use of percutaneous motor peripheral nerve stimulation (mPNS) for reduction of chronic pain related to OAk. Motor PNS is a minimally invasive treatment in which temporary percutaneous electrodes are placed near mixed nerves to stimulate motor end points to produce muscle contraction in close proximity to the painful area. Motor PNS has been shown to produce long-term pain relief after weeks of stimulation in conditions such as chronic shoulder (6-8) and back pain $(9,10)$. Though the mechanism is not known, mPNS has been hypothesized to affect chronic pain through modulation of central mechanisms that contribute to chronic pain (7), and mPNS may offer effective treatment for chronic OAkrelated pain.

From: 'MetroHealth Rehabilitation Institute, MetroHealth System, Cleveland, Ohio; ${ }^{2}$ Case Western Reserve University, Cleveland, Ohio; ${ }^{3}$ The Cleveland FES Center, Cleveland, Ohio 


\section{CASE PRESENTATION}

The patient is a 53-year-old obese male with diabetes and a body mass index of 47.1, who experienced right knee pain refractory to conservative care for 9 months. Prior treatments included physical therapy, acetaminophen, nonsteroidal anti-inflammatory drugs, intraarticular corticosteroid injections, and weight management. Physical exam revealed tenderness to palpation over the medial joint line and a positive patellar grind sign. The affected knee had full range of motion without joint laxity in any direction. Imaging revealed Kellgren \& Lawrence grade 3 knee osteoarthritis.

The intervention protocol was approved by the local institutional review board. The formal 12-week intervention protocol included lead implantation, one-week of lead stabilization, 7-weeks of mPNS treatment, and a 4-week follow-up. Outcome assessments were made at baseline, at 8 weeks (end of stimulation), and at 12 weeks. Measured outcomes include the Brief Pain Inventory-Short Form (BPI-SF), the Knee Injury and Osteoarthritis Outcome Score (KOOS), and the Patient Global Impression of Change (PGIC). The BPI-SFhas excellent psychometrics and is recommended by the Initiative on Methods, Measurement, and Pain Assessment in Clinical Trials (IMMPACT) group. Per recommendation of the developers of the BPI, BPI-SF, the "pain worst" rating, will serve as the primary response metric. The question asks patients to rate their worst pain in the prior 24 hours or prior 7 days on a 0 to 10 numeric rating scale (NRS), where " 0 " indicates "No pain" and "10" indicates "Pain as bad as you can imagine." The KOOS is a knee-specific, self-report measure that assesses the opinion of the patient about their knee and associated problems. The KOOS evaluates both short-term and long-term consequences of knee pain relative to physical function relating to daily life, sport, and recreation. This provides information related to the effect of knee pain on the patient that is complementary to the primary outcome of severity of the worst pain in the prior week. The PGIC is a self-report measure that reflects the patient's belief about the efficacy of treatment. This information is important in evaluating the effect of treatment on pain and function from the point of view of the patient who underwent the PNS treatment.

Two percutaneous leads (SPRINT Extensa ${ }^{\text {TM }}$, SPR Therapeutics, Inc., Cleveland, $\mathrm{OH}$ ), or insulated wire with distal areas of exposed electroconductive metal in contact with tissue, were placed in the medial and lateral vastus muscles of the painful knee, with the goal of stimulating femoral nerve motor end-points to produce comfortable muscle contraction. Leads were preloaded into introducers and subsequently deployed into the muscles under sterile conditions (Fig. 1). The leads were placed anatomically and with ultrasound confirmation, and appropriate placement was determined by visually confirming stimulus-evoked contraction of the target muscle. An external dual-lead stimulator was connected to the implanted leads, with the stimulator outputs a biphasic current waveform with current pulse parameter ranges that are safe for percutaneous PNS. The stimulator was set to deliver a pulse frequency of 12 $\mathrm{Hz}$, an amplitude of $20 \mathrm{~mA}$, and a pulse duration up to 200 microseconds. The pulse duration is set to produce a comfortable muscle contraction of the medial and lateral vastus muscles every 30 seconds (20 seconds of stimulation, 10 seconds off) for 6 hours per day from weeks 2-8 for a total of 294 hours. The patient controlled the stimulation via a remote control. The leads were removed at week 8 through gentle traction.

BPI results (Table 1) yielded improved scores in all categories, the most drastic being improvement in average and current pain, and pain interference. KOOS results (Table 1) revealed improvement in all categories of pain, symptoms, activities of daily living, sports and recreation, and quality of life at 8 - and 12-weeks. The PGIC at the end of stimulation was Much Improved. After the stimulation ended, the patient no longer required oral analgesics for knee pain. Stimulator compliance was 342/294 total hours. There were no serious adverse events.

\section{DISCUSSION}

Percutaneous mPNS is a minimally invasive, temporary treatment targeting mixed nerves to produce muscle contractions in close proximity to the painful region. This technology has been investigated previously and with good benefit for subacromial impingement, poststroke shoulder pain, and low back pain, with the benefit lasting well beyond the treatment period (6-10). Promising studies are ongoing for additional utilization of this methodology.

This case report demonstrates the feasibility, potential for effectiveness as a treatment of chronic pain related to OAk, and safety congruent with prior PNS studies (6-10). Due to the unique design of the percutaneous lead, tight apposition of soft tissue to the lead surface is achieved preventing microbial invasion. The open helix design also provides tension relief, which prevents 
"pistoning" of the lead relative to the skin surface. Both features translate to a minimal risk of infection when implanted for up to the approved 60 days duration, with minimal lead breakage $(11,12)$. In addition, allowing one week of healing prior to stimulation and muscle contraction propagates tissue adherence to the electrode, improving electrode/tissue juxtaposition. The resulting tight interface mitigates infection and bleeding risk (with international normalized ratio <3.0), therefore, no antibiotics or withholding of anticoagulation were implemented.

The postulated mechanism of sustained pain improvement from mPNS is central sensitizationmodulation and reversal via both direct and indirect activation of afferent proprioceptive fibers (7). While the results of this single case report are encouraging, further study is warranted.

Cost effectiveness relative to alternative care has not been established.

\section{CONCLUSION}

This case report demonstrates the feasibility of mPNS as a safe and effective treatment alternative for chronic pain related to OAk in a patient with Grade 3 knee osteoarthritis refractory to conservative care.

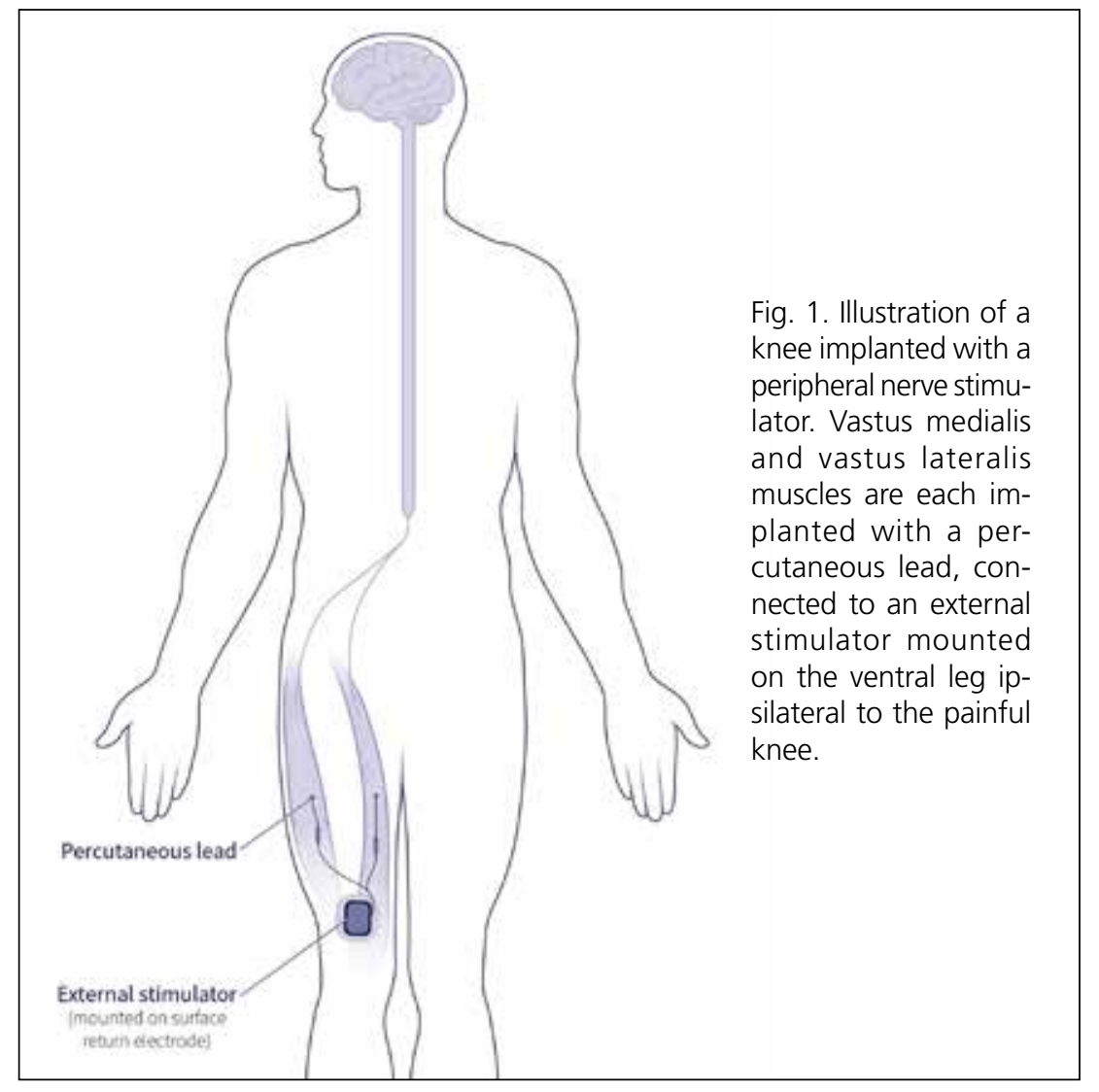

Table 1. Pain and disability ratings during course of OAk mPNS.

\begin{tabular}{|l|c|c|c|c|}
\hline Scale & Category & Baseline & 8 Weeks & 12 Weeks \\
\hline BPI-SF $^{\dagger}$ & Worse Pain & 8 & 5 & 7 \\
\hline & Average Pain & 5 & 3 & 2 \\
\hline & Current Pain & 6 & 0 & 1 \\
\hline & Pain Interference & 7 & 1 & 2 \\
\hline KOOS $^{\ddagger}$ & Pain & 11 & 78 & 83 \\
\hline & Symptom & 25 & 89 & 86 \\
\hline & ADL & 31 & 65 & 85 \\
\hline & Sport/Rec & 10 & 65 & 80 \\
\hline & QoL & 0 & 63 & 75 \\
\hline
\end{tabular}

Abbreviations: OAk: osteoarthritic knee; mPNS: motor peripheral nerve stimulation; BPI-SF: brief pain inventoryshort form; KOOS: knee injury and osteoarthritis outcome score; ADL: activities of daily living; Sport/Rec: sports and recreation; QoL: quality of life.

$\dagger$ : BPI scale range $0-10$, with 0 indicating no symptoms and 10 the worst pain possible

$\$$ : KOOS scale range $0-100$, with 100 indicating no symptoms and 0 the worse possible symptoms 


\section{REFERENCES}

1. Lawrence RC, Felson DT, Helmick CG, et al. Estimates of the prevalence of arthritis and other rheumatic conditions in the United States. Part II. Arthritis Rheum 2008;58:26-35.

2. Deshpande BR, Katz JN, Solomon DH, et al. Number of persons with symptomatic knee osteoarthritis in the US: Impact of race and ethnicity, age, sex, and obesity. Arthritis Care Res (Hoboken) 2016;68:1743-1750

3. Jevsevar DS, Brown GA, Jones DL, et al. The American Academy of Orthopaedic Surgeons evidence-based guideline on: Treatment of osteoarthritis of the knee, 2nd edition. J Bone Joint Surg Am 2013; 9595:1885-1886.

4. Zhang W, Nuki G, Moskowitz RW, et al. OARSI recommendations for the management of hip and knee osteoarthritis: Part III: Changes in evidence following systematic cumulative update of research published through January 2009. Osteoarthritis Cartilage 2010; 18:476-499.

5. Wylde V, Beswick A, Bruce J, Blom A, Howells N, GoobermanHill R. Chronic pain after total knee arthroplasty. EFORT Open Rev 2018; 3:461-470.

6. Wilson RD, Gunzler DD, Bennett ME, Chae J. Peripheral nerve stimulation compared with usual care for pain relief of hemiplegic shoulder pain: A randomized controlled trial. Am J Phys Med Rehabil 2014; 93:17-28.

7. Wilson RD, Harris MA, Gunzler DD, Bennett ME, Chae J. Percuta- neous peripheral nerve stimulation for chronic pain in subacromial impingement syndrome: A case series. Neuromodulation 2014; 17:771-776; discussion 776.

8. Chae J, Yu DT, Walker ME, et al. Intramuscular electrical stimulation for hemiplegic shoulder pain: A 12-month follow-up of a multiple-center, randomized clinical trial. Am J Phys Med Rehabil 2005; 84:832-842.

9. Cohen S, Gilmore C, Kapural L, et al. Percutaneous peripheral nerve stimulation for pain reduction and improvements in functional outcomes in chronic low back pain. Mil Med 2019; 184:537-541.

10. Gilmore CA, Kapural L, McGee MJ, Boggs JW. Percutaneous peripheral nerve stimulation (PNS) for the treatment of chronic low back pain provides sustained relief. Neuromodulation 2019; 22:615-620.

11. Ilfeld BM, Gabriel RA, Saulino MF, et al. Infection rates of electrical leads used for percutaneous neurostimulation of the peripheral nervous system. Pain Pract 2017; 17:753-762.

12. Knutson JS, Naples GG, Peckham PH, Keith WM. Electrode fracture rate and occurrences of infection and granuloma associated with percutaneous intramuscular electrodes in upper-limb functional electrical stimulation application. J Rehabil Res Dev 2003; 39:671-684. 